\title{
Affective guidance in the Iowa gambling task
}

\author{
BRANDON M. WAGAR \\ University of Victoria, Victoria, British Columbia, Canada \\ and \\ MIKE DIXON \\ University of Waterloo, Waterloo, Ontario, Canada
}

\begin{abstract}
It has been suggested that affective states can guide higher level cognitive processes and that such affective guidance may be particularly important when real-life decisions are made under uncertainty. We ask whether affect guides decisions in a laboratory task that models real-life decisions under uncertainty. In the Iowa gambling task (IGT), participants search for monetary payoffs in an uncertain environment. Recent evidence against an affective guidance interpretation of the IGT indicates a need to set a standard for what counts as evidence of affective guidance. We present a novel analysis of IGT, and our results show that participants' galvanic skin response (GSR) reflects an affective process that precedes and guides cognition. Specifically, prior to participants' knowledge of the optimal strategy, their GSRs are significantly higher when they are about to select from a bad deck, relative to a good deck, and this difference in GSR is correlated with a behavioral preference for the good deck.
\end{abstract}

The question of how much conscious control we have over our decisions is one of the most interesting and important questions of psychological research. Traditional thinking in cognitive psychology has assumed that complex decision making does not rely on, and is not necessarily influenced by, affective processes. However, recent research in psychology, behavioral economics, and neuroscience (Cohen \& Blum, 2002; Kahneman, 2003) suggests that affective processes play an important role in many decision-making processes. Specifically, it has been suggested that affective processes precede and guide cognition (Zajonc, 1980) and that affective guidance may be particularly important when real-life decisions are made in an uncertain environment (Damasio, 1994). Affect, as it is understood here, is distinct from emotion. Affect can be understood in relation to the idea of core affect (Russell, 2003). At the heart of emotion, mood, and other emotionally charged events are conscious states experienced as simply feeling good or bad. These states, called core affect, can influence perception, cognition, and behavior even though people have no direct access to these causal connections. The central question we ask is whether affect guides decisions in a laboratory task that models real-life decisions in an uncertain environment.

In the Iowa gambling task (IGT; Bechara, Damasio, Damasio, \& Anderson, 1994; Bechara, Damasio, Tranel, \& Damasio, 1997), participants search for monetary payoffs in an uncertain environment. Individuals are given a $\$ 2,000$ loan of play money and four decks of cards and are

Correspondence concerning this article should be addressed to B. M. Wagar, Department of Psychology, University of Victoria, P.O. Box 3050 STN CSC, Victoria, BC, V8W 3P5 Canada (e-mail: bmwagar@uvic.ca). instructed to make a series of card selections from any of the four decks, until they are told to stop. The participants are instructed to play so that they maximize profit on the loan. Turning each card carries an immediate reward (which is large for Decks A and B and small for Decks C and D). In addition, after turning some cards, the participants receive a punishment (which is large for Decks A and $\mathrm{B}$ and small for Decks $\mathrm{C}$ and D). Decks A and B are equivalent in terms of overall net loss, but the punishment is more frequent and of smaller magnitude for Deck A than for Deck B. Decks C and D are equivalent in terms of overall net gain, but the punishment is more frequent and of smaller magnitude for Deck C than for Deck D. The resulting combination of rewards and punishments results in an optimal strategy, so that playing from Decks A or B (bad decks) results in an overall loss, whereas playing from Decks C or D (good decks) results in an overall profit. The participants are told that they are free to switch from any deck to another at any time and as often as they wish. However, the participants are not told how many card selections must be made and are blind to the punishment schedules.

Typically, the participants adopt the optimal strategy of picking predominantly from good decks and avoiding bad decks. In fact, the participants initially start to show a physiological preference by exhibiting higher galvanic skin responses (GSRs) when they are about to select a card from a bad deck than when they are about to select a card from a good deck, even though their subjective reports fail to reveal conscious knowledge of the optimal strategy. Shortly afterward, the participants begin to demonstrate the optimal strategy in their behavioral performance (on the basis of their card selections), picking the good decks over the bad decks, even though their 
subjective reports still fail to reveal conscious knowledge of the optimal strategy. This has been interpreted as indicating that automatic processes are generating predicted outcomes in the form of affective reactions that come to guide behavior (Bechara et al., 1994; Bechara et al., 1997; Damasio, 1994). Damasio and colleagues have claimed that normal decision making in complex, uncertain environments depends on affective signals (which they call somatic markers) that warn us when important events are about to occur and guide our decisions accordingly. Damasio and colleagues stated that somatic markers can be manifested both unconsciously (when one exhibits a behavioral preference for a possible course of action in the absence of conscious knowledge) and consciously (when one exhibits a consciously accessible gut feeling or affective response that guides behavior).

It is necessary to establish to what extent affective guidance influences decision making in the IGT. Damasio and colleagues claimed that participants choose preferentially from the good decks in the IGT before being consciously aware of the optimal strategy (the relative advantage of the good decks over the bad decks). Maia and McClelland (2004) have challenged the notion that unconscious knowledge of the optimal strategy plays a role in performance on the IGT. They claim instead that participants' performance on the IGT can be explained solely by their conscious knowledge of the relative value of the decks from which they make card selections. In our view, Maia and McClelland have significantly advanced our understanding of the complexities of the IGT and have highlighted the ambiguity of the evidence previously used to support claims about affective guidance. In the present set of experiments, however, we show that a novel means of analyzing performance on the IGT can reveal that conscious knowledge in the form of affective states does, indeed, influence decision making in the IGT and that it does so prior to participants' being consciously aware of the optimal strategy. In order to understand the nature of our novel analyses, we first will consider the advances in our understanding of the IGT attributable to the work of Maia and McClelland.

Maia and McClelland (2004) focused on the status of participants' knowledge about the decks when deciding which card to select. The primary question they asked was whether the participants were truly unaware of information about the relative value of the good decks over the bad decks when they switched to the optimal strategy. By adopting a new method for probing participants' conscious knowledge about the information needed to compute the relative value of the four decks, Maia and McClelland showed that performance in the IGT could as easily be guided by conscious knowledge of the relative values of the decks as by affective influences.

In their reexamination of the IGT, Maia and McClelland (2004) raised two important issues with respect to interpreting performance on the IGT. First, they pointed out the need to reconsider Damasio and colleagues' definition of what it means to decide advantageously in the
IGT. The original definition involves contrasting the number of selections from good decks and bad decks, so that optimal performance occurs when participants select predominantly from the good decks. However, the sequence of rewards and punishments is fixed within each deck and across participants, so that early in the IGT the bad decks produce the best net outcome (i.e., if the participants have not experienced a punishment from the bad decks, the consistent $\$ 100$ reward these decks provide outweighs the $\$ 50$ reward from the good decks). Because participants can base their performance only on personal experience, Maia and McClelland cleverly pointed out that advantageous performance should consider the net outcomes experienced up to the trial on which a decision is being made, rather than the overall sequence of rewards and punishments embedded in the decks. Thus, advantageous performance is determined by calculating, for each participant and each trial, which two decks are best and which two decks are worst, on the basis of the mean net outcomes the participant has experienced with each deck up to that trial. In summary, advantageous performance on a given trial involves selecting a card from one of the two best decks (i.e., one of the two decks with the highest observed mean net outcomes up to that trial), whereas optimal performance on a given trial involves selecting a card from one of the two good decks (i.e., one of the two decks with the highest overall net outcomes across the entire game). The advantageous strategy is based on the participant's viewpoint and depends on what card selections he or she has actually made at any given point in the game. By contrast, the optimal strategy involves looking at performance from the experimenter's viewpoint. The combinations of rewards and punishments are arranged in such a way that objectively, in the long run (i.e., once enough cards have been selected), if participants choose from the good decks and avoid the bad decks, they will maximize their profits.

The second important issue Maia and McClelland (2004) highlighted is the importance of defining precisely what is meant by consciously knowing the advantageous and/or optimal strategy. To assess participants' knowledge of the optimal strategy, Bechara et al. (1997) asked them, "Tell me what you know about what is going on in this game" and "Tell me how you feel about this game" after the first 20 trials and then at 10-trial intervals. Conscious knowledge was defined ambiguously as the participants' ability to express knowledge about why, in the long run, Decks A and B were bad and Decks C and D were good. In contrast to Bechara and colleagues' vague description, Maia and McClelland specified exactly what was meant by conscious knowledge of the advantageous strategy. Knowing the advantageous/optimal strategy meant having conscious knowledge that would support the choice of one of the two best/good decks. Conscious knowledge was operationalized as knowledge that can be reported verbally. Importantly, Maia and McClelland outlined three possible levels of conscious knowledge of the advantageous/optimal strategy: 
Level 0: The participant does not have any conscious knowledge specifying a preference for one of the two best/ good decks.

Level 1: The participant has conscious knowledge specifying a preference for one of the two best/good decks but does not have conscious knowledge about the relative values of the decks that could provide a basis for that preference. Level 2: The participant has conscious knowledge specifying a preference for one of the two best/good decks and has conscious knowledge about the relative values of the decks that could provide a basis for that preference.

Maia and McClelland (2004) provided convincing evidence against Bechara et al.'s (1997) claim that participants perform optimally even when their knowledge is still at Level 0. In fact, the participants reported knowledge of the advantageous strategy more reliably than they performed advantageously (i.e., selected a card from one of the two best decks). Furthermore, when they performed advantageously, their verbal reports nearly always revealed evidence of quantitative knowledge about the outcomes of the decks that would be sufficient to guide such advantageous performance. On the basis of their results, Maia and McClelland argued that there was no decisive evidence to support the claim that unconscious processes are necessary and sufficient for the computation of the optimal strategy.

In summary, Bechara et al. (1994; Bechara et al., 1997) claimed that normal decision making in complex, uncertain environments depends on somatic markers that can be manifested both unconsciously (when one exhibits a behavioral preference for a possible course of action in the absence of conscious knowledge) and consciously (when one exhibits a consciously accessible gut feeling or affective response that guides behavior). Maia and McClelland (2004) provided convincing evidence against Bechara et al.'s claim that somatic markers can unconsciously guide behavior. Maia and McClelland further pointed out that the majority of participants had access to conscious knowledge of the advantageous strategy throughout the entire game, suggesting that performance on the IGT is driven solely by conscious, controlled processing. However, demonstrating that conscious knowledge is a possible influence on performance is not equivalent to establishing that it is the sole or even primary influence on performance. Although we agree with Maia and McClelland that unconscious processing alone does not influence card selections in the IGT, we believe that consciously accessible affective processing does influence optimal performance on the IGT prior to conscious knowledge of the optimal strategy.

The goal of the present experiment was to establish to what extent the processes that give rise to affective states, as opposed to conscious knowledge of the relative values of the decks alone, influence participants' decision making in the IGT. To what extent do participants perform optimally when their knowledge is at Level 1 (i.e., the participant has conscious knowledge specifying a preference for one of the two good decks but does not have conscious knowledge about the relative values of the decks that could provide a basis for that preference)? Maia and McClelland (2004) did not address this question directly. However, their results do call into question the necessity of assuming that Level 1 knowledge guides performance. They found that whenever participants have a preference for the best decks, the majority of participants also appear to have quantitative knowledge about the net outcomes of these decks. The challenge for the present work is to establish whether affect guides optimal performance on the IGT before participants possess conscious knowledge of the optimal strategy. Experiments 1A and 1B will provide replications of Bechara et al.'s (1997) and Maia and McClelland's methodologies for performing and analyzing the IGT, respectively. We then will present our novel analysis, which is an attempt to clarify the respective roles of affective guidance and conscious knowledge of the relative values of the decks on decision making in the IGT.

\section{EXPERIMENT 1A}

Experiment $1 \mathrm{~A}$ was a replication of the original Bechara et al. (1997) methodology for performing and analyzing the IGT.

\section{Method}

Participants. Twelve undergraduate students from the University of Waterloo participated in return for course credit.

Iowa gambling task. We used a computerized version of the IGT and monitored the participants' GSR activity. The computerized version of the gambling task was based on the original as described in Bechara et al. (1994; Bechara, Tranel, \& Damasio, 2000). In the computerized version of the gambling task, the participant saw the four decks of cards on a computer screen. The decks were labeled A, B, C, and D at the top end of each deck. Using a mouse, the participant could click on a card in any of the four decks. The computer tracked the sequence of the cards selected from the various decks. Every time the participant clicked on a deck to pick a card, the computer generated a distinct sound (similar to a casino slot machine). The face of the card appeared on top of the deck, displaying the amount of money the participant had won or lost. On the top of the computer screen was a green bar that changed according to the amount of money won or lost after each selection. A gain was indicated by a proportionate increase in the length of the green bar, and a loss was indicated by a proportionate decrease in the bar length. Once the money had been added or subtracted, the face of the card disappeared, and the participant could select another card.

The intertrial interval between the making of two consecutive card selections was set to $6 \mathrm{sec}$ to allow sufficient time for the collection of anticipatory and appraisal GSRs (see below). The total number of card selections (trials) in the experiment was set at 100 card selections. The experiment shut off automatically when the 100 selection trials were complete. However, the participant was not told in advance how many cards he/she was going to pick. To score the performance of the participant on the IGT, the number of cards picked from Decks A and B in each block of 10 cards was added; the number of cards picked from Decks C and D were added separately in each block of 10 cards.

Each deck of cards was programmed to have 40 cards. The sequence of the rewards and punishments for each deck of cards was based on the original version of this task (Bechara et al., 1994). In brief, every 10 cards from Deck A or B amounted to a gain of $\$ 1,000$, with a total loss of $\$ 1,250$ (ranging from $\$ 150$ to $\$ 350$ in Deck A and one $\$ 1,250$ loss in Deck B), bringing a net loss of $\$ 250$. On the other hand, every 10 cards from Deck C or D amounted to a 
gain of $\$ 500$, with a total loss of $\$ 250$ (ranging from $\$ 25$ to $\$ 75$ in Deck $C$ and one $\$ 250$ loss in Deck D), bringing a net gain of $\$ 250$. In summary, Decks A and B were equivalent in terms of overall net loss over trials. Similarly, Decks C and D were equivalent in terms of overall net gains. The difference was that Decks A and C had higher frequency but lower magnitude punishment. Decks B and D had lower frequency but higher magnitude punishment. Thus, Decks $\mathrm{A}$ and $\mathrm{B}$ were nonoptimal because they resulted in an overall loss in the long run, whereas Decks C and D were optimal because they resulted in an overall gain in the long run.

The computer displayed a $\$ 100$ reward every time the participant picked a card from Deck A or B and displayed $\$ 50$ when the choice was from Deck C or D. When a card corresponding to a punishment trial was picked, the computer displayed a message: "You have won X dollars, but you also have lost Y dollars" (the X amount corresponds to the magnitude of the reward, and the $\mathrm{Y}$ amount corresponds to the magnitude of the punishment). The net loss was reflected automatically on the green bar at the top of the screen. When a card corresponding to a reward-only trial was picked, the computer displayed the message, "You have won X dollars," and the gain was reflected automatically on the green bar at the top of the screen.

Note that the gambling task involved 100 selections of cards and that there were only 40 cards in each deck. Thus, it was possible to run out of cards from a given deck. When a given deck ran out of cards, the participant was instructed to stop picking from that deck and to continue choosing from the remaining decks. In reality, this situation arose very seldom. The reason is that the task was more difficult than it appeared to be. It was difficult for the participants to be sure whether to pick constantly from a given deck. Therefore, their selections were distributed among the different decks, and the decks seldom ran out of cards.

The participants' subjective reports of how they had conceptualized the game and of the strategy they had been using were assessed by interrupting the game briefly after each participant had made 20 card turns and had already encountered penalties and by asking the participant two questions: "Tell me all you know about what is going on in this game" and "Tell me how you feel about this game." The questions were repeated at 10-card intervals, and the participants wrote out their responses.

In summary, after clicking to turn each card, the participant received a reward (the amount was displayed on the screen). On some cards, the participant received both a reward and a punishment (the amounts were displayed on the screen). Clicking to turn any card from Deck A or Deck B yielded \$100; turning any card from Deck C or Deck D yielded \$50. However, the overall net value of each deck varied, because the punishment amounts were higher in the bad decks (A and B), leading to a net loss, and lower in the good decks (C and D), leading to a net gain. Thus, Decks A and B were bad, whereas Decks C and D were good.

So that they could perform the task, the participants were given the following instructions:

1. In front of you on the screen, there are four decks of cards: A, B, C, and $\mathrm{D}$.

2. I want you to select one card at a time, by clicking on the card, from any deck you choose.

3. Each time you select a card from a deck, the computer will tell you that you won some money. I won't tell you how much money you will win. You will find out along the way.

4. Every time you win, the green bar gets longer. Every so often, however, when you click on a card, the computer tells you that you won some money, but then it says that you also lost some money. I won't tell you when you will lose or how much you will lose. You will find out along the way. Every time you lose, the green bar gets shorter.

5 . You are absolutely free to switch from one deck to another any time you wish.

6. The goal of the game is to win as much money as possible and, if you find yourself unable to win, make sure you avoid losing money as much as possible.
7. I won't tell you for how long the game will continue. You must keep on playing until the computer stops.

8 . You will get this $\$ 2000$ credit (see the green bar) to start the game. At the end, we will see how much you won or lost.

9. It is important to know that computer does not make you lose money at random. However, there is no way for you to figure out when the computer will make you lose. All I can say is that you may find yourself losing money on all of the decks, but some decks will make you lose more than others. You can win if you stay away from the worst decks.

GSR recording. An automated and computerized method for collecting, measuring, and analyzing GSR data was used. Electrodes were attached to the middle and index fingers after the participant was seated in a comfortable chair in front of the computer screen. As the participant performed the IGT, GSR activity was recorded continuously and collected simultaneously on a separate computer. Each time the participant clicked the mouse and selected a card, this action was recorded as a mark on the polygram of GSR activity. Each click was registered as a selection from the specific deck that was chosen. Thus, GSRs generated in association with a specific card from a specific deck could be identified precisely on the polygram. Although the intertrial interval was set at $6 \mathrm{sec}$, in reality, the time interval between two card selections was longer, because it took a few additional seconds for the participant to decide which card to pick next. This time interval varied from trial to trial. It was, on average, $10 \mathrm{sec}$. During the 6-sec intertrial interval, the decks were displayed continuously on the screen, and the participant could ponder which deck to choose next. However, if the participant clicked the mouse to select a card during that time interval, the computer would not respond, and therefore no record was generated.

The GSRs generated during the task were divided into three categories: (1) reward GSRs, which were generated after turning cards for which there was a reward and no punishment; (2) punishment GSRs, which were generated after turning a card for which there was a reward and an immediate punishment; (3) anticipatory GSRs, which were generated previous to turning a card from any given deck (i.e., during the time period when the participant pondered which deck to choose from). The time windows for the reward and punishment GSRs were the $5 \mathrm{sec}$ immediately after the selection of a card. GSRs generated during the 5 -sec window before the next click of a card were considered anticipatory GSRs. The GSR data were acquired via an MP100WS system (BIOPAC Systems). The data were stored on a PC computer, and they were analyzed by AcqKnowledge III software for the MP100WS system. The steps involved in the quantification of anticipatory, reward, and punishment GSRs entailed the following.

The first step involved elimination of the down drift in the GSR wave. This function measured the difference (in amplitude) of two sample points that were separated by 10 samples. Then the difference was divided by the time interval between the first selected sample and the last selected sample.

The second step involved measurement of the area under the curve in the 5-sec time window after a card had been selected (for reward and punishment GSRs) and the area under the curve in the 5-sec time window immediately before a card would be clicked (for anticipatory GSRs). The area under the curve measurement was expressed in terms of amplitude units (mS) per time interval (seconds). Because the time interval was always $5 \mathrm{sec}$, we divided each area under the curve measurement by 5 . The area measurements per second $(\mathrm{mS} /$ sec) from all of the reward GSRs of the good decks were averaged. Averaging was also performed on all of the reward GSRs from the bad decks, all of the punishment GSRs from the good decks, and all of the punishment GSRs from the bad decks. Thus, for each participant, we obtained two dependent variables of reward GSRs (from good decks and from bad decks) and two dependent variables of punishment GSRs (from good decks and from bad decks).

In the case of anticipatory GSRs, the time interval varied from trial to trial, but on average, it was also $5 \mathrm{sec}$. Therefore, each area 
measurement from an individual trial was divided by its correspondent time interval. Thus, for each participant we obtained two dependent variables of anticipatory GSRs (one from the good decks and one from the bad decks).

\section{Results and Discussion}

Bechara et al. (1997) divided participants' performance in the game into a number of discrete, sequential periods characterized by the pattern of GSRs, card selections, and subjective reports. The prepunishment period occurred after all four decks had been sampled and before any losses had been encountered. The prehunch period (analogous to Maia \& McClelland's [2004] Level 0) occurred after a few losses in the bad decks had been encountered, when GSRs began to indicate the optimal strategy; yet the participants performed randomly and failed to exhibit either affective or conscious knowledge of the optimal strategy. The hunch period (analogous to Maia \& McClelland's Level 1) occurred when the participants began to perform optimally and to express a feeling that the bad decks were riskier and when they began to generate anticipatory GSRs whenever they were about to select a card from one of the bad decks and yet failed to exhibit conscious knowledge of the optimal strategy. During the conceptual period (analogous to Maia \& McClelland's Level 2), the participants expressed conscious knowledge of the optimal strategy.

The participants' card selection performance and their GSRs to good and bad decks in these four time periods are presented in Figures 1A and 1B, respectively. After sampling all four decks and before encountering any losses, (i.e., the prepunishment period), the participants selected more cards from the bad decks and did not generate significant anticipatory GSRs. After encountering a few losses in the bad decks (i.e., the prehunch period), the participants began to generate anticipatory GSRs to the bad decks (usually by Card 10). Yet, by Card 20, all indicated that they neither consciously knew nor had any feelings about the optimal strategy. By about Card 40, the participants began to perform optimally and to express a feeling that the bad decks were riskier; all generated anticipatory GSRs whenever they were about to select a card from one of the bad decks, but none exhibited conscious knowledge of the optimal strategy (the hunch period). By Card 70, all the participants expressed conscious knowledge about why, in the long run, Decks A and B were bad and Decks C and D were good (the conceptual period). All of the participants reached the conceptual period, during which they continued to prefer the good decks over the bad decks and continued to generate GSRs whenever they were about to select a card from one of the bad decks. On the basis of this method of analysis, the results of Experiment 1 indicate that the activation of affective responses (as indexed by GSRs and the participants' subjective feelings) promoted the optimal strategy before there was conscious knowledge of the optimal strategy. Specifically, during the hunch period (see Figure 1), anticipatory GSRs and card selections distinguished between good and bad decks. This suggests that the participants affectively responded to the optimal strategy well before they con- sciously knew which were the good decks and bad decks. It is important to note that Bechara and colleagues argued that affect does not make decisions per se but, rather, facilitates the efficient processing of knowledge and logic necessary for conscious decisions. Specifically, Bechara et al. (1994; Bechara et al., 1997) claimed that the pattern of anticipatory GSRs, preferential selections of cards from the good decks, and subjective expression of a hunch that the bad decks were riskier seen in the hunch period reflects automatic processes shaped by reward, punishment, and the affective state that follows them, which subsequently guide complex decision making in an uncertain environment.

\section{EXPERIMENT 1B}

Experiment 1B was a replication of Maia and McClelland's (2004) methodology for performing and analyzing the IGT.

\section{Method}

Participants. Twelve undergraduate students from the University of Waterloo participated in return for course credit.

Iowa gambling task. We used the computerized version of the IGT and monitored GSR activity as described in Experiment 1A. The only difference between Bechara et al.'s (1997) version of the IGT and Maia and McClelland's (2004) version was the questionnaire used to assess conscious knowledge. To assess participants' level of knowledge throughout the game, Maia and McClelland developed a more sensitive test of awareness in the form of the following structured questionnaire:

Q1. Rate, on a scale of -10 to +10 , how good or bad you think deck A is, where -10 means that it is terrible and +10 means that it is excellent. Q2. Okay, why did you rate deck A with...?

[Repeat questions Q1 and Q2 for decks B, C and D]

Q3. In answering the questions that follow, consider the following definitions. Your "winning amount" for a trial is the amount you won on that trial. Your "loss" on a trial is the amount you lost on that trial. Your "net result" for a trial is the amount you won minus the amount you lost on that trial. Do you understand these definitions and the differences between the three terms?

Okay, now suppose that you were to select 10 cards from deck $\mathrm{A}$.

Q3.1. What would you expect your average net result to be?

Q3.2. What would you expect your average winning amount to be?

Q3.3. In how many of the 10 trials would you expect to get a loss (not necessarily a net loss)?

Q3.4. For those trials in which you would get a loss, what would you expect the average loss to be?

[Repeat question Q3 for decks B, C and D]

Q4. Okay, now tell me, on a scale of 0 to 100 , how much you think that you know what you should do in the game in order to win as much money as possible (or, if you can't win, to avoid losing money as much as possible). 0 means that you have no idea of what you should do and feel that you still need to explore the game more and 100 means that you know exactly what you should do and have no doubts that that would be the best strategy.

Q5. Now suppose I told you that you could only select cards from one of the decks until the end of the game, but that you were allowed to choose now the deck from which you would draw the cards. Which of the four decks would you pick?

As in the method presented by Bechara et al. (1997), the participants were asked these questions after the first 20 trials and then after every 10 trials. If a participant's knowledge is at Level 1 , such knowledge should be reflected in the answers to questions Q1 and Q5. Question Q1 asks for a simple rating of each deck. The response is taken to indicate Level 1 knowledge if the deck with the highest rating is one of the two best decks according to 
the participant's experience up to that point. Question Q5 asks the participant to indicate the deck that he or she would choose if he or she could select only from that deck for the rest of the experiment. Again, the response is taken to indicate Level 1 knowledge if the participant indicates one of the two best decks. If a participant's knowledge is at Level 2, in addition to having knowledge expressing a preference for which deck is best, he or she should also have conscious knowledge about the outcomes associated with the various decks that would justify that decision. An assessment of the participants' conscious knowledge is provided by the questions in Q3. Question Q3.1 directly assesses the participants' knowledge of the expected net for each deck. The response is taken to indicate Level 2 knowledge if the participant attributes the highest expected net to one of the two best decks. Questions Q3.2-Q3.4 assess the participants' knowledge of the outcomes of each deck in terms of each deck's reward value, probability of getting a loss, and mean loss value, respectively. Note that Questions Q3.2-Q3.4 allow one to calculate the mean net that a participant should expect, on the

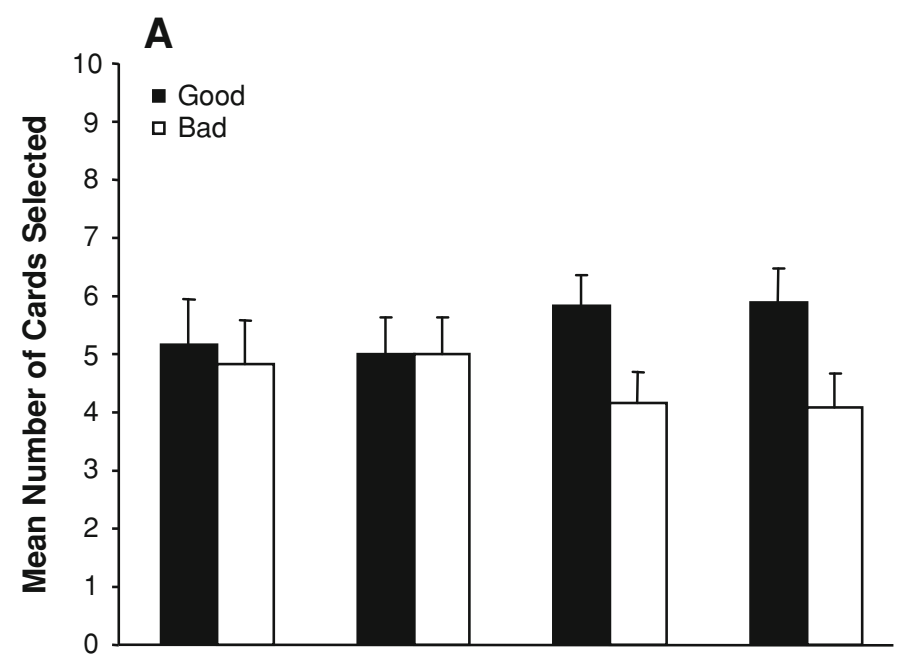

B

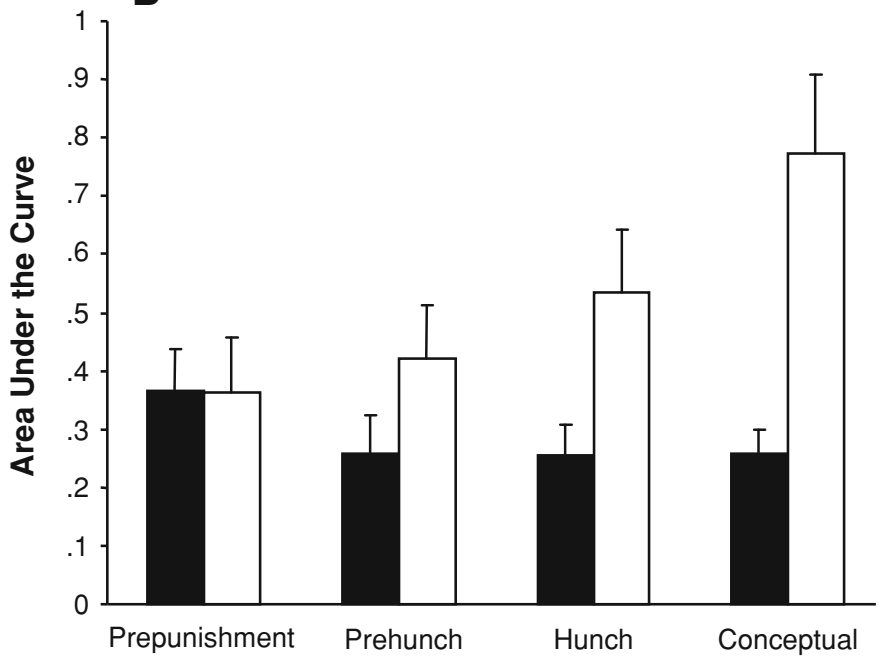

Figure 1. Performance (mean number of cards selected from the bad decks vs. the good decks) and anticipatory GSRs associated with the same cards (mean area under the curve) as a function of period. The prepunishment period occurred after all four decks had been sampled and before any losses had been encountered. The prehunch period occurred after a few losses in the bad decks had been encountered, when the participants began to generate anticipatory GSRs to the bad decks and yet neither consciously knew nor had any feelings about what was going on. The hunch period occurred when the participants began to perform optimally and to express a feeling that the bad decks were riskier; all generated anticipatory GSRs whenever they were about to select a card from one of the bad decks, but none exhibited conscious knowledge of the optimal strategy. The conceptual period occurred when the participants expressed conscious knowledge about why, in the long run, Decks $A$ and $B$ were bad and Decks $C$ and $D$ were good. 


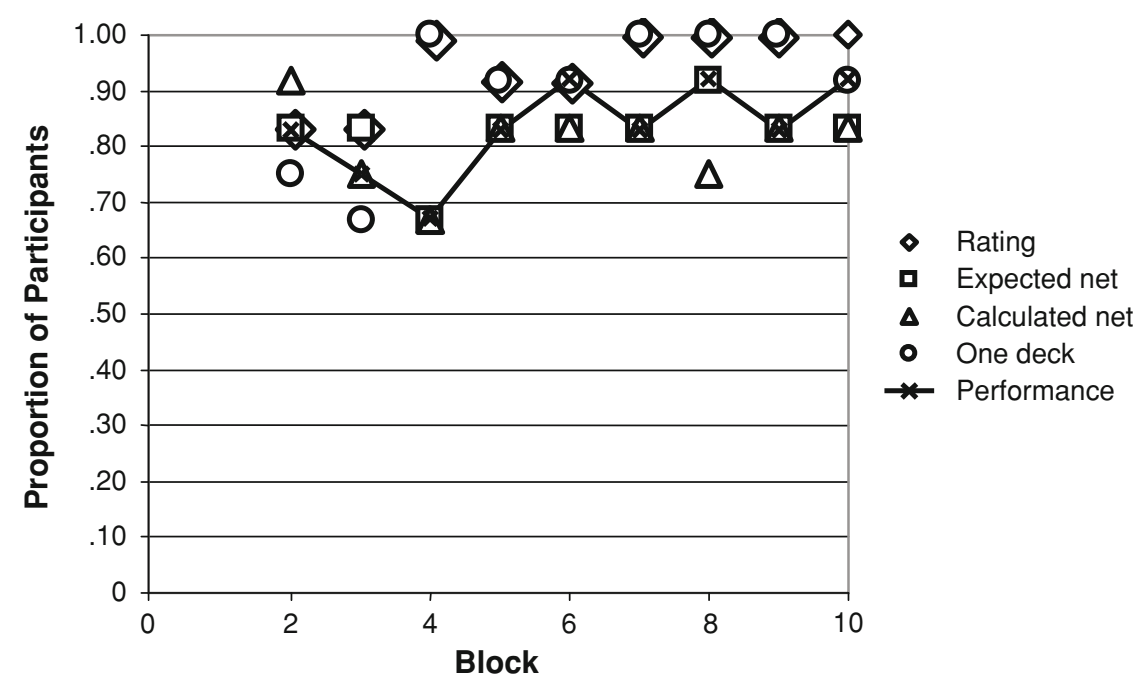

Figure 2. Participants' knowledge that one of the two best decks is the best deck, as reflected in their verbal report measures. The diamond and circle markers indicate Level 1 knowledge, and correspond to the number of participants who gave the highest rating to one of the two best decks and the number of participants who said that they would select from one of the two best decks if they could select only from one deck, respectively. The square and triangle markers indicate Level 2 knowledge. The square marker corresponds to the number of participants who gave the highest expected net to one of the two best decks, and the triangle marker corresponds to the number of participants who had the highest calculated net for one of the two best decks.

basis of his or her knowledge about the outcomes of the decks. Maia and McClelland (2004) called this value the calculated net (CN). For each question period $q$, participant $p$, and deck $d$, it is obtained from the participant's answers to questions Q3.2, Q3.3, and Q3.4 by using the following formula [in which $\mathrm{CN}(q, p, d)$ refers to the calculated net in question period $q$ for participant $p$ and deck $d$ and Q3.2( $q, p, d), \mathrm{Q} 3.3(q, p, d)$, and Q3.4( $q, p, d)$ represent the answers of participant $p$ to the corresponding questions for deck $d$ in question period $q$ ]:

$$
\mathrm{CN}(q, p, d)=\mathrm{Q} 3.2(q, p, d)+\mathrm{Q} 3.3(q, p, d) * \mathrm{Q} 3.4(q, p, d) .
$$

The responses to Questions Q3.2-Q3.4 are taken to indicate Level 2 knowledge if the highest $\mathrm{CN}$ is for one of the two best decks.

GSR recording. The automated and computerized method for collecting, measuring, and analyzing GSR data was described in Experiment 1A.

\section{Results and Discussion}

In summary, advantageous performance on a given trial involves selecting a card from one of the two best decks (i.e., one of the two decks with the highest observed mean net outcomes up until that trial), whereas optimal performance on a given trial involves selecting a card from one of the two good decks (i.e., one of the two decks with the highest overall net outcomes across the entire game).

If a participant's knowledge is at Level 1 (knowledge that he or she prefers one of the two best decks), such knowledge should be reflected in the answers to Questions Q1 (a simple rating of each deck) and Q5 (deck chosen if allowed to select only from that deck for the rest of the experiment). Again, the response is taken to indicate Level 1 knowledge if the participant's preferences indicate one of the two best decks. If a participant's knowledge is at Level 2 (quantitative knowledge that the net outcome is highest for one of the two best decks), in addition to having knowledge expressing a preference for which deck is best, he or she should also demonstrate conscious knowledge about the outcomes associated with the various decks that would justify that decision. An assessment of a participant's conscious knowledge is provided by Question Q3.1 (expected net) and Questions Q3.2-Q3.4 (CN).

Figure 2 shows how many participants showed evidence of Level 1 and Level 2 knowledge of the advantageous strategy in the different verbal report measures, as well as how many participants behaved advantageously (solid line). As can be seen, for the majority of participants, in every period in which the participants' knowledge was probed, the verbal report measures demonstrate conscious knowledge about the outcomes associated with the various decks and conscious knowledge about the advantageous strategy. In fact, the tendency is for knowledge of the advantageous strategy to be more evident in the verbal report measures than in performance. Figure 2 shows that all of the participants who performed advantageously on a given block showed Level 1 knowledge of the advantageous strategy in their answers to Questions Q1 (circle) and Q5 (diamond). Figure 2 further shows that, in the majority of cases, the participants who performed advantageously also showed knowledge of the advantageous strategy in both quantitative measures of Level 2 knowledge: the expected net (square) and the $\mathrm{CN}$ (triangle).

The first question of interest is whether there were any participants who performed advantageously and demon- 
strated Level 1 knowledge (knowledge that they preferred one of the two best decks) but did not show evidence of conscious knowledge of the advantageous strategy on either measure of Level 2 knowledge (quantitative knowledge that the net outcome was highest for one of the two best decks). This can be seen in Figure 2, where the circle and diamond markers reflect a higher percentage of participants' knowledge than do the square and the triangle markers. In Maia and McClelland's (2004) data, 1 participant (out of 19,5\%) performed advantageously and demonstrated Level 1 knowledge in several (three) question periods in which neither Level 2 measure reflected knowledge of the advantageous strategy. In our data, 4 participants (out of 12,33\%) performed advantageously and demonstrated Level 1 knowledge during at least one of the question periods, and 2 participants $(16 \%)$ performed advantageously and demonstrated Level 1 knowledge in several (three) question periods, in which neither Level 2 measure reflected knowledge of the advantageous strategy. Thus, our results are quite similar to those obtained by Maia and McClelland.

In addition, as was seen in Maia and McClelland's (2004) data, it is also apparent in the figure that on several blocks, a small number of participants failed to show evidence of knowledge of the advantageous strategy on at least one of these two measures (i.e., the square and the triangle do not overlap). These participants cannot be definitively classified as being at Level 1 or 2, because they demonstrated inconsistent conscious knowledge in the quantitative Level 2 measures. However, the data show that in the overwhelming majority of cases in which the participants behaved advantageously, they exhibited Level 2 knowledge in both the reported net and the CN.

It is important to note that participants at Level 2 do not necessarily keep track throughout the game of the numerical estimates that they provide when answering the questions in Q3 that provide the expected net and the CN. Participants may produce such estimates only at the time the questions are asked, possibly by sampling relevant exemplars from memory. It cannot be assumed that performance is based directly on this type of quantitative information. Even though Maia and McClelland (2004) and our replication of their results in Experiment 1B have shown that participants' conscious knowledge is sufficient to explain their advantageous performance, the extent to which the participants actually based their performance on this conscious knowledge at the time of choice remains an open question. The fact that the participants' verbal reports demonstrated conscious knowledge when asked to do so does not imply per se that such conscious knowledge played a causal role in their actual decisions. Nevertheless, if, whenever they perform advantageously, participants can show evidence of knowledge of the advantageous strategy in their answers to the Level 2 quantitative measures, one can conclude that they have consciously accessible knowledge that provides a sufficient basis for such advantageous performance.

The primary question Maia and McClelland (2004) asked was whether participants were truly unaware of in- formation about the relative value of the good decks over the bad decks when they began to perform optimally. Although their method of analysis does not explicitly show the point at which the good decks become the best decks (i.e., the point at which the optimal strategy becomes advantageous), it is intuitive from their analysis that when participants begin to perform optimally, they have consciously accessible knowledge that provides a sufficient basis for such performance.

When reflecting on the significance of the studies of Bechara et al. (1994; Bechara et al., 1997) and the study of Maia and McClelland (2004) for us, the primary question boils down to whether the consciously accessible knowledge of participants undertaking the IGT represents Level 1 or Level 2 knowledge.

The pattern of results seen in Bechara et al.'s (1997) data, as well as in our replication of Bechara et al., in Experiment $1 \mathrm{~A}$, suggests that the point at which the optimal strategy becomes advantageous is associated with Level 1, rather than Level 2, knowledge. During Bechara and colleagues' hunch period, none of the participants exhibited conscious knowledge of the optimal strategy (indicative of Level 2 knowledge). Despite this, the participants stated that they preferred the good decks, began to select cards optimally, and generated anticipatory GSRs whenever they were about to select a card from one of the bad decks. Our replication confirms each of these findings and suggests that when the data are analyzed following the methods of Bechara et al., the point at which participants switch to the optimal strategy is associated with Level 1 (preference) knowledge, rather than with Level 2 (quantitative) knowledge about each deck.

Maia and McClelland's (2004) findings are less clear with respect to this issue. However, there are several indicators that suggest that participants may not have initially been consciously aware of the optimal strategy when it became advantageous. First, in both Maia and McClelland's data and our replication in Experiment 1B, a small percentage of participants performed advantageously yet demonstrated only Level 1 knowledge in several question periods in which neither Level 2 measure reflected knowledge of the advantageous strategy. This is the clearest evidence that at least for these people (33\% in our replication), the point at which the optimal strategy became advantageous might have been associated with Level 1, rather than Level 2, knowledge. Unfortunately, without knowing exactly when the optimal strategy became advantageous, it is impossible to know for sure. Second, there were, on some blocks, a small number of participants who performed advantageously and demonstrated Level 1 knowledge but showed conscious knowledge of the advantageous strategy on just one of the two measures of Level 2 knowledge (i.e., the expected net and the CN did not coincide). Maia and McClelland attributed this to noisy estimates; however, because of this inconsistency, these participants cannot be definitively classified as being at Level 1 or 2. Finally, Maia and McClelland also included a question that assessed the participants' overall confidence in their understanding of the game (Question 


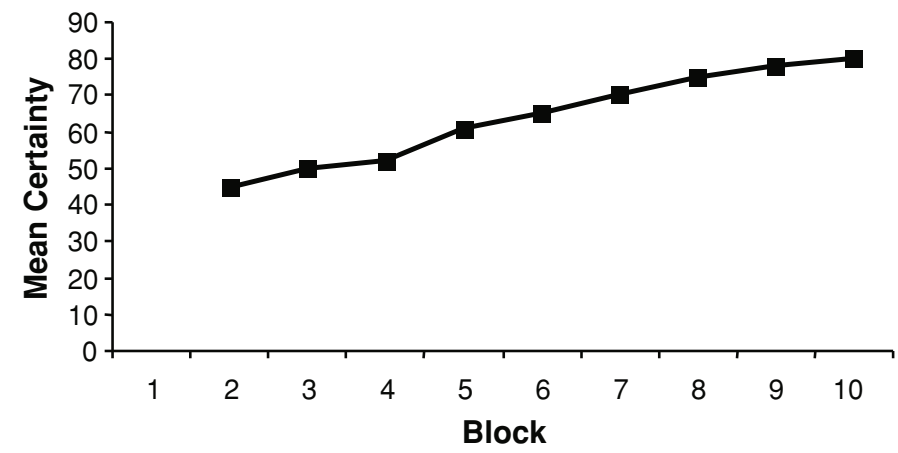

Figure 3. Mean certainty reported by the participants as a function of block.

Q4). Maia and McClelland claimed that this question was meant to address issues unrelated to those discussed in this article, and so the data were presented in the online supplementary information. An analysis of this question in both Maia and McClelland's data and our data (see Figure 3) revealed that the participants' certainty about what they should do in the game increased gradually as the game progressed. Thus, although the participants were able to consciously report the quantitative information characteristic of Level 2 knowledge, suggesting that conscious knowledge alone could provide a sufficient basis for advantageous performance, they were unsure of this information until relatively late in the game. In summary, the existence of the hunch period and, to a lesser extent, the ambiguities in Maia and McClelland's results open up the possibility that optimal performance during the point at which the optimal strategy became advantageous might have been associated with Level 1, rather than Level 2, knowledge. However, a new type of data analysis is required to address this possibility.

To what extent do affective processes (Level 1), as opposed to conscious knowledge of the optimal strategy (Level 2), influence participants' decision making in the IGT? The strongest evidence that affective processes guide performance on the IGT comes from Bechara et al.'s (1997) finding that during the hunch period, in which the participants failed to exhibit conscious knowledge of the optimal strategy, the participants began to perform optimally (on the basis of the cards they selected) and to exhibit affective guidance (on the basis of their subjective reports and anticipatory GSRs) whenever they were about to select a card from one of the bad decks. Maia and McClelland (2004) suggested that the finding that participants have far more conscious knowledge of the advantageous strategy than Bechara et al. (1997) claimed raises the possibility that consciously accessible knowledge could provide a sufficient basis for such advantageous performance. However, the fact that the participants' verbal reports indicated conscious knowledge when they were asked to do so does not per se imply that such conscious knowledge played a primary causal role in their actual card selections. In order to understand the rela- tive influence of affective guidance and conscious control on performance on the IGT, it is necessary to include dependent measures of each. Unfortunately, Maia and McClelland did not include GSR as a measure in their experiment. Despite this omission, Maia and McClelland argued, in their discussion section, that the GSRs reflect affective responses that are elicited by processes that are consciously accessible and that also can guide behavior. They argued that there is no need to think that the GSRs reflect unconscious processing that plays a primary causal role in guiding participants' behavior. The question we are asking here is whether the affective response associated with the GSRs represents Level 1 or Level 2 knowledge. Because we included GSR as a dependent measure in our replication of Maia and McClelland's results, we are able to address this question more directly. We now will present a novel analysis of Experiments 1A and 1B in which an attempt will be made to clarify the respective roles of affect and conscious knowledge of the relative values of the decks on decision making in the IGT.

\section{REANALYZING EXPERIMENTS 1A AND 1B}

Because the novel analysis presented here is independent of the questionnaire given to the participants (i.e., it requires only data from the participants' card selections and their GSRs), we were able to apply the analysis to both Experiments 1A and 1B.

\section{Card Selections}

As was previously mentioned, good and bad decks refer to the overall outcomes associated with each deck across the entire game. At each stage of the game, however, the overall outcomes associated with the decks will depend on how many cards a given participant has chosen from a given deck (i.e., a participant cannot know that a given deck is bad if he or she selected only three cards from this deck and none of the three cards contained a punishment). To take this into account, we first located, for each participant, three separate stages based on their experience with each deck, describing the information that each deck is capable of conveying. We calculated the observed net 
outcome (the total value of a given deck, as a function of its rewards and punishments, experienced up to that point) for each of the decks at each of the 100 card selections made throughout the experiment. Stage 1 included those card selections during which the observed net outcome for the two bad decks exceeded the observed net outcome for the two good decks. That is, the two bad decks were the advantageous decks. Stage 2 included those trials during which the observed net outcomes for one of the two bad decks and one of the two good decks were advantageous. Stage 3 included those trials during which the observed net outcome for the two good decks exceeded the observed net outcome for the two bad decks. The beginning of the last stage marks the point at which the optimal strategy became advantageous (i.e., the two good decks were advantageous and the two bad decks were disadvantageous). From the participant's point of view, at Stage 1, the best decks are the bad decks (because he or she has not encountered any punishments in selecting cards from these decks, but only high rewards). Here, the advantageous strategy is to choose only from these bad decks. Note that at this point in the game, the advantageous strategy is completely misaligned with the optimal strategy, which the participant will come to learn by the end of the game. At Stage 2, with more card selections, from the participant's point of view, the two best decks comprise one of the good decks and one of the bad decks. Here, the advantageous strategy is only partially aligned with the optimal strategy that the participant will come to know. At Stage 3, the two best decks are the two good decks, and the advantageous strategy is to sample from these decks and avoid the bad decks. At this point, the advantageous strategy of the participant is now completely aligned with the optimal strategy.

Maia and McClelland (2004) stated that if a participant has conscious knowledge of the advantageous strategy, in addition to having knowledge expressing a preference for which deck is best, he or she should also have conscious knowledge about the outcomes associated with the various decks that would justify that decision. Because the participant can base his or her performance only on personal experience, advantageous performance must be based on the net outcomes of each of the decks experienced up to the trial in which a decision is being made, rather than on the overall sequence of rewards and punishments embedded in the decks. Thus, the point at which the optimal strategy becomes advantageous (i.e., the point at which the participant shifts from Stage 2 into Stage 3) marks the earliest stage in the experiment at which a participant could possibly have conscious knowledge of the optimal strategy.

An independent samples $t$ test showed that the point at which the optimal strategy became advantageous did not differ between the two experiments $[t(22)=0.07, p=$ .94]. As can be seen in Figure 4A, this point occurred between Trial 34 and Trial 56, with a median of 44 for Experiment 1A (the replication of Bechara et al., 1997). As can be seen in Figure 5A, this point occurred between Trials 30 and 59, with a median of 44 for Experiment 1B (the replication of Maia \& McClelland, 2004). This shows that the point at which the optimal strategy becomes advantageous (marking the earliest stage in the experiment at which a participant could possibly have conscious knowledge of the optimal strategy) is uninfluenced by the manner in which the participants' subjective reports are obtained (the vague questions of Bechara et al., or the more extensive and detailed questions of Maia \& McClelland) and occurs between Block 4 (Card Selections 31-40) and Block 6 (Card Selections 51-60).

Following the procedures of Bechara and colleagues, we then subdivided the 100 card selections into 10 blocks of 10 cards each. For each block, we counted the number of selections from the bad decks (A and B) and the number of selections from the good decks (C and D). A 2 (experiment) $\times 2$ (deck) $\times 10$ (block) ANOVA on the total number of card selections failed to reveal a significant three-way interaction $[F(9,198)=1.263, p=.26]$. Thus, the overall pattern of card selections did not differ between experiments. Figures $4 \mathrm{~B}$ and $5 \mathrm{~B}$ present the number of cards selected from good and bad decks as a function of block for Experiments 1A and 1B, respectively. At a cursory glance it can be seen that as the task progressed, the participants gradually shifted their preference toward the good decks (C and D) and away from the bad decks (A and $\mathrm{B})$, as is reflected in the pattern of their card selections.

A 2 (deck) $\times 10$ (block) ANOVA on the total number of card selections revealed a significant interaction $[F(9,99)=6.63, p<.001]$ in Experiment $1 \mathrm{~A}$. The same interaction was significant in Experiment 1B $[F(9,99)=$ 17.6, $p<.001]$. Post hoc Bonferroni-corrected paired sample $t$ tests comparing the number of card selections from good and bad decks during each block revealed that the participants began selecting preferentially from the good decks during Block 4 (Card Selections 31-40) in both Experiment $1 \mathrm{~A}[t(11)=9.4, p<.001]$ and Experiment $1 \mathrm{~B}[t(11)=10.4, p<.001]$.

Interestingly, this shows that the participants exhibited a behavioral preference for the good decks over the bad decks within Block 4 (Card Selections 31-40) despite the fact that for the majority of the participants $(58 \%$ in $1 \mathrm{~A}, 66 \%$ in $1 \mathrm{~B})$, the point at which the optimal strategy became advantageous did not occur until Block 5 (Card Selection 41 or later). That is, the participants showed a behavioral preference for the good decks over the bad decks (evidence of the optimal strategy) before the optimal strategy could possibly have become advantageous (and consequently, before the participants could have conscious knowledge of the optimal strategy). At first glance, this finding seems contradictory. The participants selected cards in accordance with the optimal strategy (i.e., selected more cards from the good than from the bad decks), despite the fact that these decks were not consistently the advantageous decks. Yet at the same time, the participants performed advantageously throughout the entire experiment. This seemingly contradictory finding becomes clear when one considers that on any given trial, advantageous performance involves selecting a card from one of the two best decks (i.e., one of the two decks with 
the highest observed net outcomes up to that trial). The key to this contradiction and one of the crucial analyses that demonstrates affective guidance in the IGT involves the participants' performance at Stage 2.

As was described above, there were three distinct stages with respect to the possible combinations of good and bad decks when it was considered which two decks were advantageous: (1) Both of the bad decks were advantageous, (2) one of the good decks and one of the bad decks were advantageous, and (3) both of the good decks were advantageous (i.e., the optimal strategy was advantageous). Very early in the game, both of the bad decks were advantageous. As the game progressed, the participants inevitably experienced punishments, and the advantageous decks switched to one of the good decks and one of the bad decks. Eventually, the participants thoroughly experienced the reward and punishment schedule embedded within the four decks, so that both of the good decks became advantageous. The seemingly contradictory finding that the participants showed evidence of the optimal strategy before it could possibly have become advantageous, yet still performed advantageously, is explained when one examines the participants' card selections during the second stage, when one of the good decks and one of the bad decks were advantageous.
A 2 (experiment) $\times 3$ (stage) repeated measures ANOVA examining participants' preference for good decks over bad decks (taken as the net score for each stage, where net score equals the number of selections from the good decks minus the number of selections from the bad decks) failed to show a significant interaction $[F(2,44)=0.1]$, demonstrating once again that the pattern of card selections did not differ between experiments. The critical question concerns the participants' preference during the second stage. Stage 2 occurred before the participants could possibly have had conscious knowledge of the optimal strategy, because only one of the best decks was good the other best deck was a bad deck. If performance is driven solely by Level 2 knowledge, the frequency of advantageous card selections from the two best decks (one of which is a good deck and other is a bad deck) during this stage should be equivalent (differ only by chance). Because the participants' card selections during this stage occurred before they could have had conscious knowledge of the optimal strategy, there should not have been a preference for one of the best decks over the other. However, in both experiments, on those trials on which the participants performed advantageously when one of the good decks and one of the bad decks were advantageous, all the participants showed a preference for the advantageous good deck over the ad-
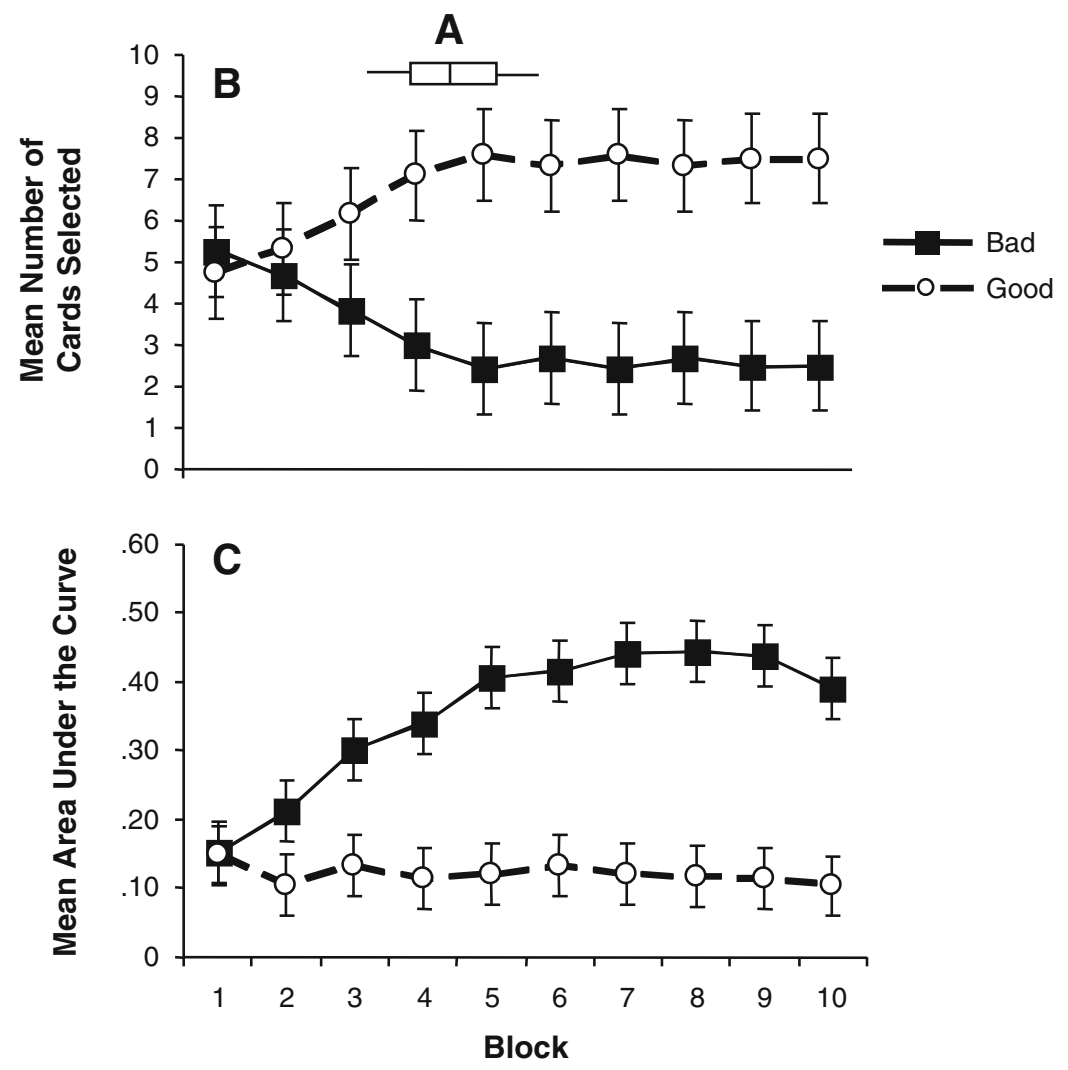

Figure 4. (A) The point at which the optimal strategy became the advantageous strategy, (B) performance (mean number of cards selected from the bad decks vs. the good decks), and (C) anticipatory GSRs associated with the same cards (mean area under the curve), as a function of block, for Experiment $1 \mathrm{~A}$. 
vantageous bad deck. In Experiment 1A, the participants selected from the advantageous good deck an average of $69 \%$ of the time during this period (preferences ranged from $58 \%$ to $80 \%, S D=6 \%$ ). In Experiment $1 \mathrm{~B}$, the participants selected from the advantageous good deck an average of $71 \%$ of the time during this period (preferences ranged from $58 \%$ to $81 \%, S D=7 \%$ ). Thus, embedded within their preference for advantageous over disadvantageous decks, the participants in both experiments demonstrated a preference for good over bad decks. Critically, this preference, which is consistent with the optimal strategy, occurred before the participants could have had conscious knowledge of the optimal strategy. In our view, the only way this could have occurred is if affective guidance influenced the participants' performance on the IGT.

\section{Anticipatory GSRs}

We now will turn to an examination of the anticipatory GSRs, which are presumed to index automatic processes that give rise to affective states. Figures $4 \mathrm{C}$ and $5 \mathrm{C}$ show the average anticipatory GSRs associated with the good and the bad decks for each of the 10 blocks for Experiments $1 \mathrm{~A}$ and $1 \mathrm{~B}$, respectively. Concordant with the card selections, as the task progressed, the participants gradually shifted their preference away from the bad decks, as is reflected in the larger anticipatory GSRs to these decks.

A 2 (experiment) $\times 2$ (deck) $\times 10$ (block) repeated measures ANOVA on the GSRs failed to reveal a significant three-way interaction $[F(9,198)=0.73, p=.68]$, demonstrating that the overall pattern of GSRs did not differ between the experiments. A 2 (deck) $\times 10$ (block) ANOVA on the GSRs revealed a significant interaction $[F(9,99)=$ $12.261, p<.001]$ in Experiment 1A. The same interaction was significant in Experiment 1B $[F(9,99)=4.016, p<$ $.001]$. Post hoc Bonferroni-corrected paired sample $t$ tests comparing the mean area under the curve for anticipatory GSRs to good and bad decks during each block revealed that the participants began showing a physiological preference for the good decks during Block 3 in both Experiment 1A $[t(11)=5.33, p<.001]$ and Experiment $1 \mathrm{~B}[t(11)=$ $3.24, p=.008]$. Thus, the participants in both experiments showed larger anticipatory GSRs to bad decks than to good decks before they began to demonstrate a behavioral preference for good decks in their card selections.

A 2 (experiment) $\times 3$ (stage) repeated measures ANOVA examining the participants' preference for good decks over bad decks (taken as the mean GSR to the bad decks minus the mean GSR to the good decks) failed to yield a significant interaction $[F(2,44)=0.4]$, showing
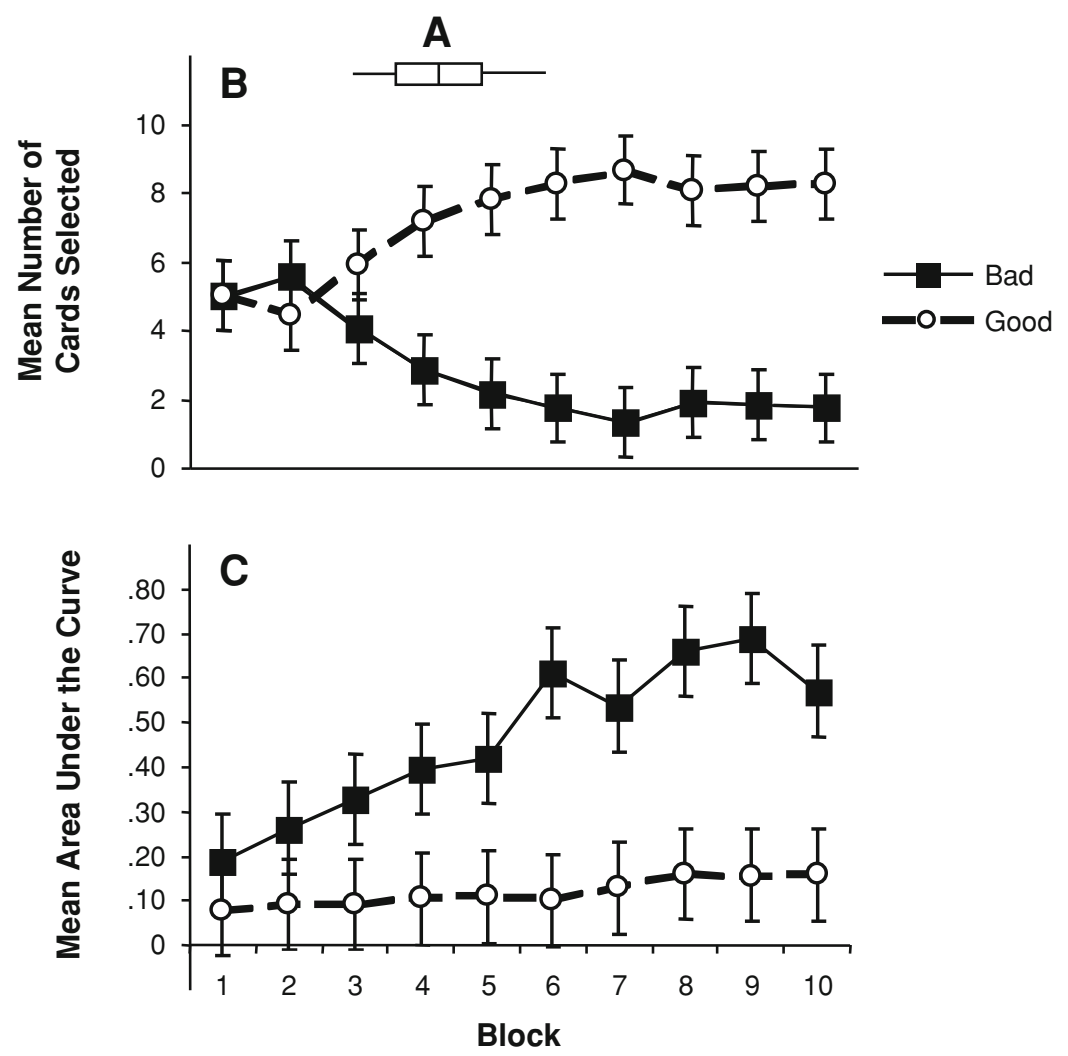

Figure 5. (A) The point at which the optimal strategy became the advantageous strategy, (B) performance (mean number of cards selected from the bad decks vs. the good decks), and (C) anticipatory GSRs associated with the same cards (mean area under the curve), as a function of block, for Experiment $1 \mathrm{~B}$. 
again that the overall pattern of GSRs did not differ between experiments. Maia and McClelland (2004) argued, in their discussion section, that the GSRs seen in Bechara and colleagues' studies reflected affective responses that are elicited by processes that are consciously accessible and that also can guide behavior. The question we are asking here is whether the consciously accessible knowledge associated with the GSRs represents Level 1 or Level 2 knowledge.

The key to addressing this question lies once again in the patterns of GSRs across the three distinct stages of the IGT. Specifically if participants show GSR preferences for the good deck over the bad deck in Stage 2 (where both of these decks are advantageous), this would demonstrate that GSRs separate good decks from bad decks before participants can possibly know the optimal strategy (hence, before they can possibly have reached Level 2). In Experiment 1A, the participants generated larger GSRs to bad decks than to good decks (mean difference $=0.34 \mathrm{mS}$ / sec) during this critical period than they did when both of the bad decks were advantageous (mean difference $=$ $0.14 \mathrm{mS} / \mathrm{sec})[t(11)=3.31, p=.007]$. In Experiment 1B, the participants generated larger GSRs to bad decks than to good decks (mean difference $=0.284 \mathrm{mS} / \mathrm{sec}$ ) during this key period than they did when both of the bad decks were advantageous (mean difference $=0.14 \mathrm{mS} / \mathrm{sec}$ ) $[t(11)=2.68, p=.02]$. Thus, the participants in both experiments demonstrated, embedded within their preference for advantageous over disadvantageous decks, a preference for good over bad decks. Critically, this GSR preference, which is consistent with the optimal strategy, occurred before the participants could possibly have had conscious knowledge of the optimal strategy.

To address the extent to which GSRs are indicative of affective responses that can guide behavior prior to conscious knowledge of the optimal strategy, we correlated the participants' GSRs with their card selections during those trials on which one of the good decks and one of the bad decks were advantageous (i.e., during Stage 2, during which conscious knowledge could not possibly have been influencing performance). We correlated the difference between good and bad deck anticipatory GSRs during this period with the difference in card selections from good and bad decks. There was a strong correlation $(r=.77$ in $1 \mathrm{~A}, r=.73$ in 1B) between the participants' preference for good over bad decks in terms of their card selection performance and their anticipatory GSRs. Specifically, the participants who generated larger GSRs to the bad than to the good deck selected more cards from the advantageous good deck than from the advantageous bad deck during those trials on which conscious knowledge could not possibly have been influencing performance.

\section{GENERAL DISCUSSION}

In summary, the consciously accessible knowledge associated with GSR activity in the IGT reflects affective guidance. GSRs and, soon afterward, card selections separated good versus bad decks prior to the point at which conscious knowledge of the optimal strategy could possibly have been influencing performance. The fact that GSRs separated earlier than card selections and that GSRs were correlated with card selections during Stage 2 shows that affective guidance, as opposed to conscious knowledge of the relative values of the decks, influences participants' decisions in the IGT.

An unexpected question that arises concerns the nature the information that is driving affective guidance in the IGT. Conscious reasoning is driven by the outcomes experienced for each of the decks. However, given that affective guidance precedes conscious reasoning, what is driving affective guidance? The higher anticipatory GSR for the bad decks than for the good decks has traditionally been interpreted as reflecting covert knowledge of the optimal strategy (Bechara et al., 1997). However, given that the GSRs separate before participants have experienced enough card selections to possibly know what the optimal strategy is, the traditional interpretation needs revision.

One possible interpretation is that the higher uncertainty associated with the bad decks is responsible. In the IGT, the bad decks also have the highest variance (the amount of money both gained and lost is much greater for bad than for good decks). Tomb, Hauser, Deldin, and Caramazza (2002) have shown that in a modified version of the IGT in which the good decks have the highest variance, participants exhibit larger GSRs when they are about to select from the good decks, even though they continue to perform optimally. This finding suggests that the higher GSRs in the IGT are related to the higher variance; they are riskier choices. It may be that affective guidance in the IGT is driven by the risk associated with a given deck, which can be established much earlier (i.e., with fewer card selections) than conscious knowledge of the relative values of the decks required to know the optimal strategy. According to this interpretation, the fact that affective guidance in the IGT follows the optimal strategy occurs only because the bad decks are riskier, rather than affect's reflecting the optimal strategy prior to conscious knowledge of the relative values of the decks.

The idea that decisions under uncertainty involve multiple processes and that these processes can react differently to different kinds of information is not novel. For example, Huettel and colleagues (Huettel, Song, \& McCarthy, 2005) demonstrated distinct neural mechanisms subserving different forms of uncertainty resolution. The notion that affective guidance reacts to different information than does conscious knowledge of the optimal strategy is important because it highlights the potential for affective misguidance. As in the IGT, where the pattern of risk is correlated with, but not causally linked to, the optimal strategy, realworld situations may arise in which the affective component of our decisions is derived from different information than are our conscious strategies. To the extent that the affective experience is driven by information that is at odds with the optimal strategy, affective guidance may actually impair our decisions. In fact, recent work by Shiv and colleagues (Shiv, Loewenstein, Bechara, Damasio, \& Damasio, 2005) has shown exactly such a situation. 
Ultimately, more work is required to fully understand the relationship between affective guidance and conscious knowledge of the optimal strategy. However, although the nature of affective guidance in the IGT remains unclear, the present results show that affective guidance does, indeed, influence participants' performance on the IGT.

\section{REFERENCES}

Bechara, A., Damasio, A. R., Damasio, H., \& Anderson, S. W. (1994). Insensitivity to future consequences following damage to human prefrontal cortex. Cognition, 50, 7-15.

Bechara, A., Damasio, H., Tranel, D., \& Damasio, A. R. (1997). Deciding advantageously before knowing the advantageous strategy. Science, 275, 1293-1295.

Bechara, A., Tranel, D., \& Damasio, H. (2000). Characterization of the decision-making deficit of patients with ventromedial prefrontal cortex lesions. Brain, 123, 2189-2202.

Cohen, J. D., \& BLum, K. I. (2002). Reward and decision. Neuron, 36, 193-198.

DAMASIO, A. R. (1994). Descartes' error: Emotion, reason, and the human brain. New York: Putnam.
Huettel, S. A., Song, A. W., \& McCarthy, G. (2005). Decisions under uncertainty: Probabilistic context influences activation of prefrontal and parietal cortices. Journal of Neuroscience, 25, 3304-3311.

Kahneman, D. (2003). A perspective on judgment and choice: Mapping bounded rationality. American Psychologist, 58, 697-720.

Maia, T. V., \& McClelland, J. L. (2004). A reexamination of the evidence for the somatic marker hypothesis: What participants really know in the Iowa gambling task. Proceedings of the National Academy of Sciences, 101, 16075-16080.

RUSSELL, J. A. (2003). Core affect and the psychological construction of emotion. Psychological Review, 110, 145-172.

Shiv, B., Loewenstein, G., Bechara, A., Damasio, H., \& Damasio, A. R. (2005). Investment behavior and the negative side of emotion. Psychological Science, 16, 435-439.

Tomb, I., Hauser, M., Deldin, P., \& Caramazza, A. (2002). Do somatic markers mediate decisions on the gambling task? Nature Neuroscience, 5, 1103-1104.

ZAJONC, R. B. (1980). Feeling and thinking: Preferences need no inferences. American Psychologist, 35, 151-175.

(Manuscript received November 10, 2005; revision accepted for publication May 30, 2006.) 